\title{
Performance indicators for wheeled robots traversing obstacles
}

\author{
William Nowac, Francisco González, Sadhbh MacMahon, József Kövecses
}

This is a post-peer-review, pre-copyedit version of an article published in IEEE Robotics and Automation Letters. The final authenticated version is available online at: http://dx.doi.org/10.1109/LRA. 2020 . 2974431.

(C2020 IEEE. Personal use of this material is permitted. Permission from IEEE must be obtained for all other uses, in any current or future media, including reprinting/republishing this material for advertising or promotional purposes, creating new collective works, for resale or redistribution to servers or lists, or reuse of any copyrighted component of this work in other works.

\begin{abstract}
An important element of wheeled robot operations on uneven and unstructured terrain is the ability to overcome obstacles. In this paper we deal with a part of this obstacle negotiation problem. We particularly investigate the ability of a wheeled robot, originating from its mechanical design, to successfully negotiate an obstacle. The work reported primarily investigates how the mechanism topologies and the resulting mass and inertia distributions influence obstacle negotiation. The kinematics of the obstacle and ground contact is described using the variables that represent the degrees of freedom of the articulated mechanical system of the robot; this enables the study of the effect of the robot topology on the contact dynamics. Based on this we develop a dynamics formulation that allows us to propose performance indicators to characterize the ability of the wheeled robot to overcome obstacles. This formulation accounts for the unilateral nature of interaction between robot, obstacle and ground. We illustrate the work with simulation and experimental results.
\end{abstract}

Keywords: Wheeled Robots, Field Robots, Contact Modelling, Kinematics, Motion Control 


\section{Introduction}

Mechanical performance is an essential element for the operation of all vehicles, but it becomes particularly important for off-road vehicles that operate on challenging uneven terrains. Mechanical performance is interpreted here as the general capability of the articulated mechanical system to carry out the objectives of the required operation.

For the operation of a wheeled robot in unstructured environments there are generally two important basic, required operational primitives. The first is to move on flat, soft terrain, and the second is to climb and traverse obstacles. In this paper we particularly analyze the obstacle negotiation mode of wheeled robots.

In the obstacle negotiation task the key element is the description of the interaction between the vehicle, the obstacle, and the rest of the terrain. Characterizing how the mechanical design of the robot can influence this interaction can be a core element in making design and operational decisions. The designer has control over the mechanical properties of the robot, but not on the obstacle or the terrain.

From the point of view of obstacle interaction modelling we can distinguish between dynamic and static interactions. In dynamic interactions the mass and inertia properties of the robot play an important role. This is particularly relevant at the onset of the interaction, when the wheels establish contact with the obstacle; the way how interaction forces are developed can be significantly influenced by the mass and inertia properties of the articulated mechanical system of the robot. The nature of these interaction forces is key in the mobility and ability of the robot to overcome the obstacle.

In static interaction the mass and inertia properties do not necessarily play a critical role. In such a case the nature of the interaction forces is mostly controlled by the kinematic transformation that relates actuator efforts and reaction forces, and also by the structural stiffness properties of the robot. Such interaction can typically represent slow-moving rovers in the later sustained phases of wheel-object contact during obstacle traversing.

The wheel-obstacle contact conditions are dominant factors in determining the quality of the obstacle negotiation manoeuvre. Little has been reported in the literature on the dynamics of this operation. A few papers report work based on quasi-static models where the wheelterrain contact points are analyzed to assess how capable the rover would be to climb a slope 
or an obstacle [1], [6]. Shifting of the centre of mass can also be used as a control strategy to influence the contact forces [12]. The concept of minimum friction coefficient that is required to maintain the stability of the rover on uneven terrain is discussed in [13]. However, none of these studies address the detailed representation and analysis of the wheel-obstacle contact, the effect of the unilateral nature of interaction of the other wheels with the terrain, and the dynamics of the robot-obstacle-terrain interaction. Dynamics can be important here even for slow motion as obstacle negotiation is associated with impacts due to the change in system topology, which causes energy and momentum transfer.

In this paper, a set of indicators to characterize the obstacle climbing manoeuvre of wheeled robots are put forward. These indicators make it possible to determine whether having a nonzero translational velocity immediately prior to contacting the obstacle is beneficial for completing the manoeuvre or if, conversely, the robot should be brought to a stop before starting to climb. The proposed indicators are easy to evaluate and based only on kinematic and kinetic quantities, thus avoiding the need to determine or estimate contact properties such as stiffness and friction, which can be difficult to obtain accurately. These indicators can also be used as guidelines for the design and operation of wheeled robots, predicting their behaviour in the negotiation of obstacles and other impact scenarios without the need to run forward-dynamics simulations that can be potentially expensive in terms of time and computational requirements. The validity of these indicators was confirmed with simulations and experiments.

\section{Dynamics modelling}

In this section, a dynamics model for a wheeled robot is presented. Based on this model, an impulse-momentum formulation is derived to analyze the time interval when a wheel of the robot initially contacts an obstacle. A method to decompose the dynamics into a subspace of constrained motion and a subspace of admissible motion is then used to formulate indicators used to predict the performance of a wheeled robot when crossing obstacles based on its mechanical design and kinematics. In order to form this decomposition, it is necessary to handle the unilateral contact problem to determine first which wheels will carry load. This leads to a formulation that requires the solution of a linear complementarity problem (LCP). Finally, three performance indicators are proposed: the admissible velocity, and the kinetic energies in the 
admissible and constrained subspaces.

\subsection{Dynamics model}

We will primarily deal with aspects of dynamic interaction in this paper. The mechanical system of the robot can be represented by an $n$-degree of freedom model, which represents the mechanical elements of the robot and the interconnections/articulations between them. We consider that this model represents a minimum degree of freedom model with respect to the internal articulations and joints of the robot, i.e., the related constraints are implicitly considered by selecting a minimum set of generalized coordinates and velocities. On the other hand, the interaction forces developed at the wheel-ground and wheel-obstacle interfaces are explicitly represented. Such a model can generally be formulated as

$$
\mathbf{M} \dot{\mathbf{v}}+\mathbf{c}(\mathbf{v}, \mathbf{q})=\mathbf{f}+\mathbf{A}_{w}^{\mathrm{T}} \boldsymbol{\lambda}_{w}+\mathbf{A}_{o}^{\mathrm{T}} \boldsymbol{\lambda}_{o}
$$

where $\mathbf{M}$ is the $n \times n$ mass matrix, $\mathbf{v}$ and $\mathbf{q}$ represent the $n \times 1$ arrays of generalized velocities and coordinates, respectively, $\mathbf{c}$ is the $n \times 1$ array of Coriolis and centrifugal effects, $\boldsymbol{\lambda}_{w}$ and $\boldsymbol{\lambda}_{o}$ are the $g \times 1$ and $m \times 1$ arrays of the friction and normal reaction force components, developed at the wheel-ground and wheel-obstacle interfaces, respectively, and $\mathbf{A}_{w}$ and $\mathbf{A}_{o}$ are the related, $g \times n$ and $m \times n$, Jacobian matrices, and finally $\mathbf{f}$ is the $n \times 1$ array of other external and internal generalized forces acting on the robot. These other generalized forces include for example the internal actuation forces/torques and structural stiffness representation of the robot and gravity. For the examples given in the paper, as a simplification, all joints were considered frictionless. However, friction can be included in the generalized force terms and it would not influence the decomposition concept and how the nature of momentum and energy distributions is affected by the mechanism topology and the wheel-object contact. We consider that the wheel-ground interactions give rise to $g$ force components, and the wheel-obstacle contact is represented by $m$ force components. We assume that modelling the robot components as rigid bodies gives a reasonably accurate representation as we primarily intend to study dynamic interaction where mass and inertia properties play the dominant role and those can be well-captured with the rigid body model. 


\subsection{Impulse-momentum formulation}

The left-hand side of Eq. (1) can be reformulated as

$$
\mathbf{M} \dot{\mathbf{v}}+\mathbf{c}=\dot{\mathbf{p}}+\mathbf{d}
$$

where

$$
\mathbf{p}=\frac{\partial T}{\partial \mathbf{v}}=\mathbf{M v}
$$

is the generalized momentum of the system, and $T$ represents the kinetic energy. As the generalized velocities, $\mathbf{v}$, can also include non-holonomic components, the general interpretation of the modified nonlinear inertial term $\mathbf{d}$ is possible through Hamel's equations [10] for example, or can also be symbolically written as $\mathbf{d}=\mathbf{c}-\dot{\mathbf{M}} \mathbf{v}$. With this, Eq. (1) can be rewritten as

$$
\dot{\mathbf{p}}+\mathbf{d}(\mathbf{v}, \mathbf{q})=\mathbf{f}+\mathbf{A}_{w}^{\mathrm{T}} \boldsymbol{\lambda}_{w}+\mathbf{A}_{o}^{\mathrm{T}} \boldsymbol{\lambda}_{o}
$$

and for an arbitrary time interval $\left[t_{1}, t_{2}\right]$ we can write the impulse-momentum equations as

$$
\mathbf{p}_{2}-\mathbf{p}_{1}=\overline{\mathbf{f}}-\overline{\mathbf{d}}+\mathbf{A}_{w}^{\mathrm{T}} \overline{\boldsymbol{\lambda}}_{w}+\mathbf{A}_{o}^{\mathrm{T}} \overline{\boldsymbol{\lambda}}_{o}
$$

where $\mathbf{p}_{1}$ and $\mathbf{p}_{2}$ represent the generalized momentum at times $t_{1}$ and $t_{2}$, respectively, $\overline{\mathbf{f}}=$

$\int_{t_{1}}^{t_{2}} \mathbf{f} d t, \overline{\mathbf{d}}=\int_{t_{1}}^{t_{2}} \mathbf{d} d t, \overline{\boldsymbol{\lambda}}_{w}=\int_{t_{1}}^{t_{2}} \boldsymbol{\lambda}_{w} d t$, and $\overline{\boldsymbol{\lambda}}_{o}=\int_{t_{1}}^{t_{2}} \boldsymbol{\lambda}_{o} d t$ are the impulses of the related generalized forces. If the duration of the time interval $[t 1, t 2]$ is small compared to the time scale of the system dynamics, then it can be assumed that the system configuration does not change during it. Therefore, matrices $\mathbf{A}_{w}$ and $\mathbf{A}_{o}$ can be considered constant from the point of view of determining the impulses. Eq. (4) represents how the impulse of the different forces can affect the change of momentum in a certain time period, and vice versa, how a change in momentum can affect the forces. Based on Eq. (4) we can also see that the impulse associated with each set of generalized forces contributes to the total change in momentum, and these contributions can be superimposed on each other to obtain the total change. To characterize the obstacle negotiation abilities of the wheeled robot we can consider that interval $\left[t_{1}, t_{2}\right]$ is selected so that $t_{2}$ is a time point just after the contact with the obstacle is established, and $t_{1}$ is the time shortly before the contact onset. When contact is established with the obstacle at a certain time instant the velocity components that represent the relative motion between the wheels and the obstacle 
reduce to zero. This instant is a particularly important selection for time $t_{2}$; we will consider this in the following. We are primarily looking at how the mass and inertia properties can affect the interactions of the robot with the obstacle and the ground. Therefore, in Eq. (4) the $\bar{f}$ and $\overline{\mathbf{d}}$ impulses can be neglected, i.e., we assume that the impulse caused by actuators and external forces, $\overline{\mathbf{f}}$, as well as the impulse originating from the nonlinear inertial terms, $\overline{\mathbf{d}}$, are considered negligible in the short $\left[t_{1}, t_{2}\right]$ period compared to the other terms in Eq. (4). These assumptions are common when studying impact problems [3]. Therefore, with these considerations, Eq. (4) can be reduced to

$$
\mathbf{p}_{2}-\mathbf{p}_{1}=\mathbf{A}^{\mathrm{T}} \overline{\boldsymbol{\lambda}}
$$

where

$$
\mathbf{A}=\left[\begin{array}{l}
\mathbf{A}_{o} \\
\mathbf{A}_{w}
\end{array}\right] \quad \text { and } \quad \boldsymbol{\lambda}=\left[\begin{array}{l}
\overline{\boldsymbol{\lambda}}_{o} \\
\overline{\boldsymbol{\lambda}}_{w}
\end{array}\right]
$$

Further expanding the momentum terms based on Eq. (2) we can obtain

$$
\mathbf{M} \Delta \mathbf{v}=\mathbf{A}^{\mathrm{T}} \overline{\boldsymbol{\lambda}}
$$

where $\Delta \mathbf{v}=\mathbf{v}\left(t_{2}\right)-\mathbf{v}\left(t_{1}\right)$ and $\mathbf{M}$ is interpreted for the configuration associated with time $t_{1} \cdot{ }^{1}$

\subsection{Linear complementarity problem}

We consider the onset of contact with the obstacle to determine how the mechanical structure of the robot can influence its tendency and ability to climb the obstacle. The modes of motion that can be constrained by the contact with the obstacle and the wheel-ground interaction can be represented at the velocity level as

$$
\mathbf{A}_{o} \mathbf{v}=\mathbf{u}_{o} \quad \text { and } \quad \mathbf{A}_{w} \mathbf{v}=\mathbf{u}_{w}
$$

\footnotetext{
${ }^{1}$ This involves some approximation as this is exact for the representation of $\mathbf{p}_{1}$, however, it assumes that the configuration, and with that $\mathbf{M}$, are not changing much during the investigated time interval.
} 
where $\mathbf{u}_{o}$ and $\mathbf{u}_{w}$ are $m \times 1$ and $g \times 1$ arrays that include the velocity components associated with the directions to be constrained by the obstacle contact and the wheel-ground contacts, respectively.

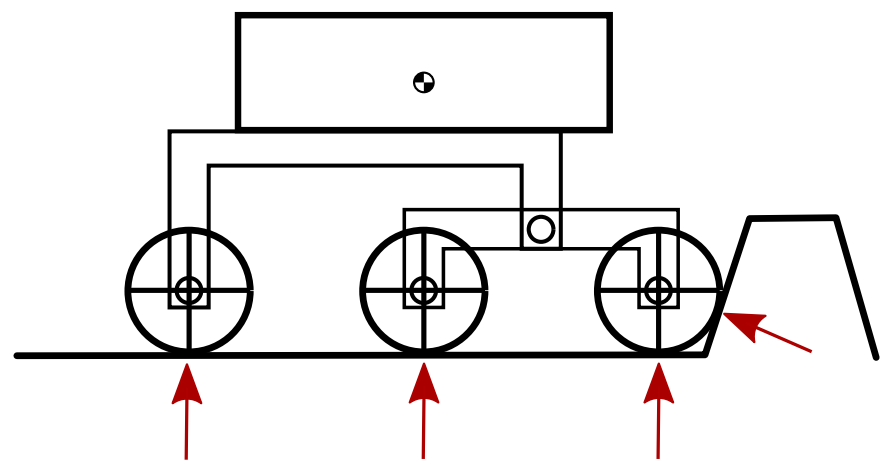

Figure 1: A planar model of a six-wheeled robot contacting an obstacle. The arrows represent the normal directions of the contacts.

Fig. 1 illustrates the case when these constrained modes include the directions normal to the obstacle. Using Eq. (8) the related kinematic equation can also be written as

$$
\mathbf{A} \Delta \mathbf{v}=\Delta \mathbf{u}
$$

where $\Delta \mathbf{u}=\left[\begin{array}{ll}\Delta \mathbf{u}_{o}^{\mathrm{T}} & \Delta \mathbf{u}_{w}^{\mathrm{T}}\end{array}\right]^{\mathrm{T}}$ with $\Delta \mathbf{u}=\mathbf{u}\left(t_{2}\right)-\mathbf{u}\left(t_{1}\right)$. Considering the solution of Eq. (7) for $\Delta \mathbf{v}$ and substituting that into Eq. (9) we can obtain

$$
\mathbf{A M}^{-1} \mathbf{A}^{\mathrm{T}} \overline{\boldsymbol{\lambda}}=\Delta \mathbf{u}
$$

which is interpreted in the constrained subspace and plays the key role to determine the impulse distributions. Our intention is to determine which wheel contacts are active. Eq. (10) on its own does not constitute a solvable set for $\bar{\lambda}$ and $\Delta \mathbf{u}$ as it does not contain enough information about the dynamic behaviour that is of interest. This additional information has to be added via giving constitutive relations for the impulses and the related velocity changes. As we are not necessarily looking for the exact values of the impulses, but rather to determine whether or not a contact is active we will assume the constitutive relation that all contacts can be considered equally stiff, which, in turn, from the point of view of determining the nature of force distributions, can be equivalently represented with constraints and complementarity relations in the form of $\Delta \mathbf{u} \geq \mathbf{0}$, $\overline{\boldsymbol{\lambda}} \geq \mathbf{0}$ and $\Delta \mathbf{u}^{\mathrm{T}} \overline{\boldsymbol{\lambda}}=0$, which can also be written in the compact form

$$
\mathbf{0} \leq \Delta \mathbf{u} \perp \bar{\lambda} \geq \mathbf{0}
$$


Eqs. (10) and (11) represent a LCP for $\bar{\lambda}$ and $\Delta \mathbf{u}$. If the matrix $\mathbf{A M}^{-1} \mathbf{A}^{\mathrm{T}}$ is invertible then this LCP is solvable. The LCP can generally be solved in a straightforward way using direct, pivoting methods [7].

\subsection{Subspaces of admissible and constrained motion}

It can be shown that based on Eq. (8) the linear space that represents all possible modes of motion of the system can be decomposed to two subspaces: the subspace of constrained motion, and the subspace of admissible motion. Projector operators that can decompose the kinematic and dynamic quantities to the spaces of constrained and admissible motions can be constructed as

$$
\mathbf{P}_{c}=\mathbf{M} \mathbf{A}_{a c t}^{\mathrm{T}}\left(\mathbf{A}_{a c t} \mathbf{M}^{-1} \mathbf{A}_{a c t}^{\mathrm{T}}\right)^{-1} \mathbf{A}_{a c t} \quad \text { and } \quad \mathbf{P}_{a}=\mathbf{I}-\mathbf{P}_{c}
$$

where $\mathbf{P}_{c}$ and $\mathbf{P}_{a}$ are the $n \times n$ projector matrices to the subspaces of constrained and admissible motions, respectively [8]. The norm and orthogonality in the related linear space is defined in the mass-orthogonal sense. $\mathbf{A}_{a c t}$ is the subset of $\mathbf{A}$ which contains all constraints that would have non-zero impulse. In other words, $\mathbf{A}_{\text {act }}$ should only include the rows that are associated with contacts that carry load. The effective, active set of wheels are the ones where the related components of $\bar{\lambda}$ are positive and the associated elements in $\Delta \mathbf{u}$ are zero. to determine which wheel contacts satisfy these conditions, the LCP must be solved. Once this solution is known then we can determine what wheels, i.e., which rows, need to be considered to assemble the active submatrix, $\mathbf{A}_{a c t}$, of $\mathbf{A}$ in Eq. (6).

\subsection{Performance indicators}

The use of projectors $\mathbf{P}_{a}$ and $\mathbf{P}_{c}$ completely decouples the momentum, velocity, and kinetic energy to constrained and admissible parts. For the velocity of the system,

$$
\mathbf{v}=\mathbf{P}_{c} \mathbf{v}+\mathbf{P}_{a} \mathbf{v}=\mathbf{v}_{c}+\mathbf{v}_{a}
$$


where $\mathbf{v}_{c}=\mathbf{P}_{c} \mathbf{v}$ and $\mathbf{v}_{a}=\mathbf{P}_{a} \mathbf{v}$ are the constrained and admissible components, respectively, for the momentum the decomposition is

$$
\mathbf{p}=\mathbf{P}_{c}^{\mathrm{T}} \mathbf{p}+\mathbf{P}_{a}^{\mathrm{T}} \mathbf{p}=\mathbf{p}_{c}+\mathbf{p}_{a}
$$

where $\mathbf{p}_{c}=\mathbf{P}_{c}^{\mathrm{T}} \mathbf{p}$ and $\mathbf{p}_{a}=\mathbf{P}_{a}^{\mathrm{T}} \mathbf{p}$ are the constrained and admissible components of the momentum, and for the kinetic energy

$$
T=\frac{1}{2} \mathbf{v}^{\mathrm{T}} \mathbf{M} \mathbf{v}=T_{c}+T_{a}=\frac{1}{2} \mathbf{v}_{c}^{\mathrm{T}} \mathbf{M} \mathbf{v}_{c}+\frac{1}{2} \mathbf{v}_{a}^{\mathrm{T}} \mathbf{M} \mathbf{v}_{a}
$$

where $T_{c}=1 / 2 \mathbf{v}_{c}^{\mathrm{T}} \mathbf{M} \mathbf{v}_{c}$ and $T_{a}=1 / 2 \mathbf{v}_{a}^{\mathrm{T}} \mathbf{M} \mathbf{v}_{a}$ are the constrained and admissible parts of the kinetic energy, respectively. We note that there are no coupling terms in this decomposition; the quantities associated with dynamic behaviours in the subspaces are completely separated. These decomposed quantities can be used to establish performance indicators that link the mechanical design and related parameters of the robot to the desired operational behaviour. The design of the system is reflected in the mass matrix and the matrices that make up the projector operators as well as the full $\mathbf{A}_{o}$ and $\mathbf{A}_{w}$ matrices. These are analytic functions of the design parameters and the mechanism topologies selected for the articulated mechanical system of the robot.

From these, three performance indicators were selected: the constrained part of the kinetic energy prior to the establishment of the contact between the front wheel and the obstacle, $T_{c}^{-}$, the admissible part of the kinetic energy, $T_{a}^{-}$, and the admissible velocity, $\mathbf{v}_{a}$.

\subsection{1 $T_{c}^{-}$}

It was shown in [5] and [9] that the kinetic energy associated with the space of constrained motion can be used as a measure, alternative to the contact force, to represent the intensity of the establishment of contact. $T_{c}^{-}$can be used to set limits on the speed at which a wheeled robot approaches an obstacle to avoid damage. It can also be used to design wheeled robots that will encounter lower impact forces during various manoeuvres. 


\subsection{2 $T_{a}^{-}$}

The kinetic energy in the admissible subspace is the portion of energy that remains unaffected by the impact with the obstacle, whereas the kinetic energy in the constrained subspace may be dissipated and/or transferred to rebound after the impact. $T_{a}^{-}$may be compared to the gravitational potential energy required to climb over an obstacle to gauge how much momentum can help the robot traverse an obstacle.

\subsubsection{Admissible velocity}

Here we will particularly consider how the admissible velocity component, $\mathbf{v}_{a}$, is related to the ability of the robot to successfully start to negotiate the obstacle. The constrained and admissible velocities can also be seen as modes of motion where it is not the magnitude that is important, but rather the ratios among the components, which will represent the nature of motion in the subspace considered. The admissible mode describes how the robot will react to the contact onset with the obstacle, i.e., how it would tend to move on its own after contacting the obstacle. By visualizing $\mathbf{v}_{a}$ using computer animations, it is possible to identify which modes of motion hinder progress when going over the obstacle and which modes facilitate progress. Examples of these modes of motion will be shown later in the results section in Figs. 5 - 8. In cases where the mode of motion hinders progress, the robot should be stopped right before it contacts the obstacle, whereas if the mode of motion facilitates progress, the robot should approach the obstacle at a speed that is below the speed at which the robot would sustain damage from the impact, but above zero, so that its momentum can assist in climbing the obstacle and potentially reduce energy consumption. To represent the effect of $\mathbf{v}_{a}$ in a single scalar value we introduced an admissible velocity denoted by $v_{a}$. This scalar indicator directly represents the magnitude of the component of the admissible velocity of the wheel in contact with the obstacle along the direction tangent to the obstacle surface. This can be constructed as

$$
\mathbf{B} \mathbf{v}_{a}=v_{a}
$$

where $\mathbf{B}$ is a $1 \times n$ matrix that describes how the tangent direction can be decomposed using the parameterization selected for the generalized velocities of the robot. 


\section{Results}

The three indicators introduced in the previous section were tested in the obstacle climbing manoeuvre of a six-wheeled robot model.

\subsection{Small wheeled robot example}

A six-wheel, triple bogie configuration like the one shown in Fig. 1 was chosen as test example. In this arrangement, the chassis is connected via passive revolute joints to port, starboard, and rear bogies. Each bogie mounts two wheels. This configuration is frequently used in exploration rovers, e.g., [2], [11].

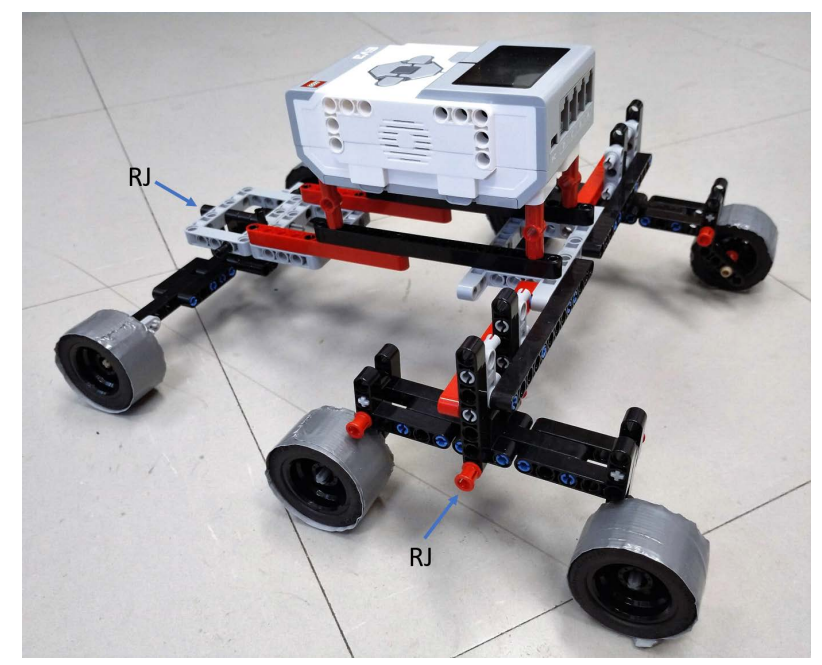

Figure 2: Model for experimental validation. $\mathrm{RJ}=$ revolute joint.

A prototype (Fig. 2) was built using Lego Mindstorms components to verify the ability of the selected indicators to capture the behaviour during the climbing manoeuvre. The prototype had a wheelbase of $26 \mathrm{~cm}$; its front bogie was $13 \mathrm{~cm}$ long, its total height was $15 \mathrm{~cm}$; its wheel radius was $2.2 \mathrm{~cm}$ and its mass was $0.6 \mathrm{~kg}$.

\subsection{Computation of indicators}

Using Eqs. (13), (15) and (16), the indicators were evaluated for different values of the obstacle angle $\theta$ at a forward speed of $0.1 \mathrm{~m} / \mathrm{s}$, immediately before contacting the obstacle. Results for 
the front wheels of the robot are shown in Fig. 3 and results for the middle wheels are shown in Fig. 4.

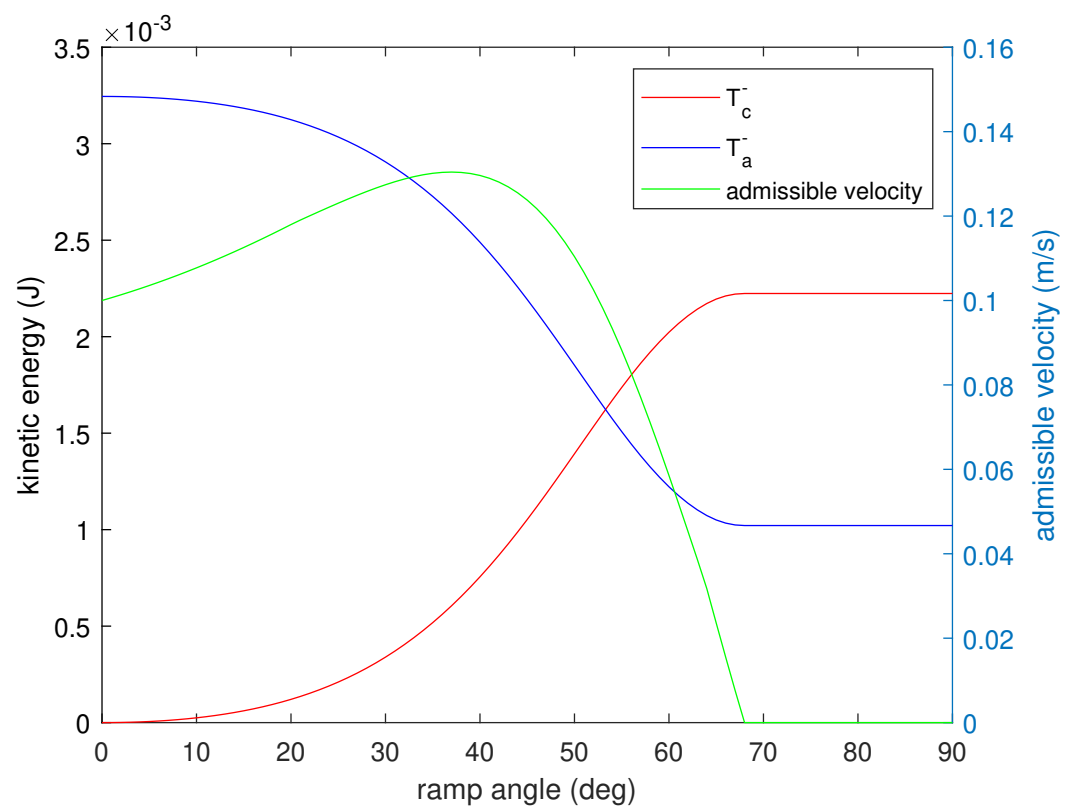

Figure 3: Impact indicators for the front wheels vs. the obstacle angle, $\theta$.

\subsection{Interpretation}

The admissible velocity $v_{a}$ in the graphs represents the magnitude of the velocity component in the subspace of admissible motion of the front and middle wheels, respectively. This scalar quantity is representative of the admissible velocity vector $\mathbf{v}_{a}$. The best way to visualize and understand the admissible velocity is to look at animations showing the robot moving with that velocity. Figs. 5 and 6 show snapshots of these animations. The top image shows the robot right before it contacts the obstacle and the bottom image shows the robot, after a certain time, if it were to move at a constant velocity equal to $\mathbf{v}_{a}$. From these figures, two modes of motion were identified. When $\theta=50^{\circ}$ the robot climbs the obstacle but when $\theta=65^{\circ}$ the robot tends to tip forward instead of climbing the obstacle. In Fig. 3, at $\theta=50^{\circ}, v_{a}=0.13 \mathrm{~m} / \mathrm{s}$ i.e., the velocity of the front wheel tangent to obstacle is slightly greater than its initial velocity which indicates that the front wheel will go up the obstacle. At $\theta=65^{\circ}, v_{a}=0.02 \mathrm{~m} / \mathrm{s}$ which indicates a much more reduced tendency to climb the obstacle.

The admissible velocity curve for the front wheels shows that the admissible velocity increases to a maximum at $\theta=38^{\circ}$, it then drops to zero at $\theta=68^{\circ}$. This indicates that contacting 


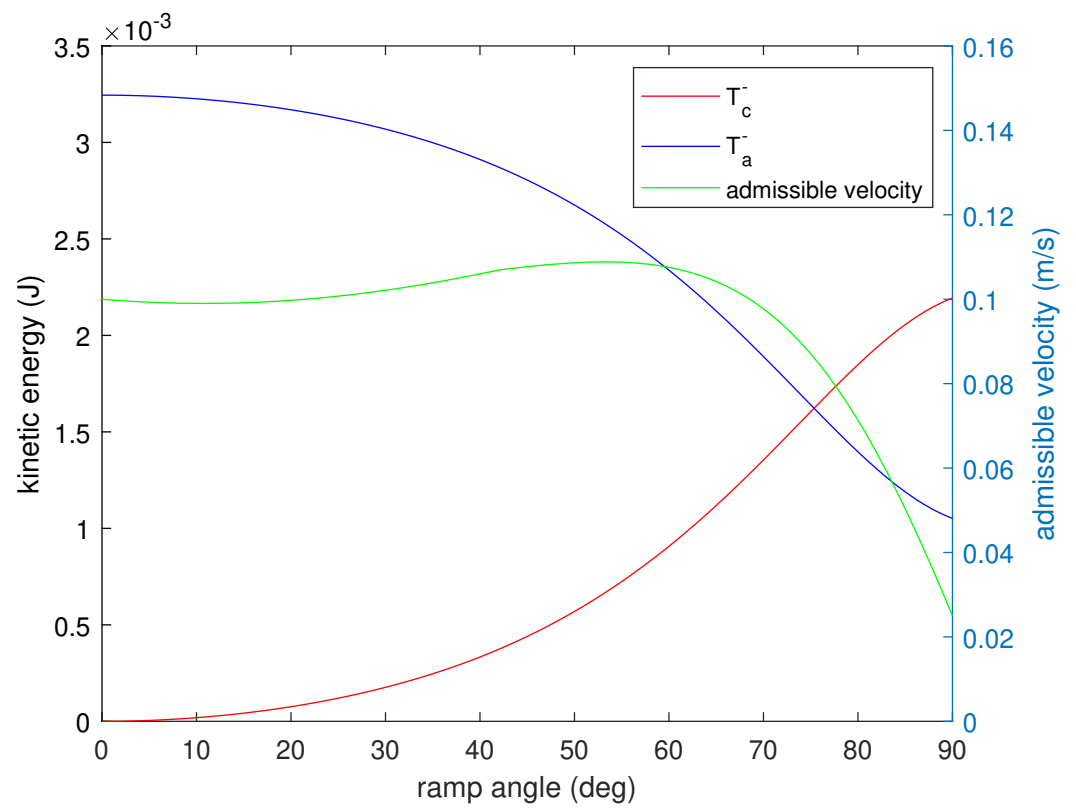

Figure 4: Impact indicators for the middle wheels vs. the obstacle angle, $\theta$.

an obstacle that has an angle less than $68^{\circ}$ with a certain speed can help overcome it because the robot will tend to climb. On the other hand, if the angle is more than $68^{\circ}$, the robot should be stopped before attempting to climb because its kinetic energy will not contribute to overcoming the obstacle, but to increase the impact force instead. In the case of the middle wheels, the admissible velocity curve reaches a maximum much later at $\theta=62^{\circ}$ and then decreases rapidly to $0.026 \mathrm{~m} / \mathrm{s}$ at $\theta=90^{\circ}$. This indicates for example, that for a $70^{\circ}$ obstacle, it would be advisable to stop the robot before the front wheel contacts the obstacle but not after the front wheels have started to climb and the middle wheels contact it.

The kinetic energy in the constrained subspace, $T_{c}^{-}$, indicates impact intensity. For the front wheels, this quantity increase with the ramp angle until it reaches a plateau at $\theta=68^{\circ}$. This is the same point at which the admissible velocity reaches zero. For the middle wheels, $T_{c}^{-}$ increases at a slower rate. This indicator can be used to determine maximum speeds when contacting obstacles to avoid damage or if it is safe to drive down a step obstacle.

Finally, the kinetic energy in the admissible subspace, $T_{a}^{-}$, is the counterpart to $T_{c}^{-}$. In order to climb up a certain obstacle, the robot must overcome gravity. Comparing $T_{a}^{-}$with the difference in gravitational potential energy at the top and bottom of the obstacle gives an indication of how much momentum will help going over the obstacle.

The example presented was selected because it clearly illustrates the concepts of admissible 


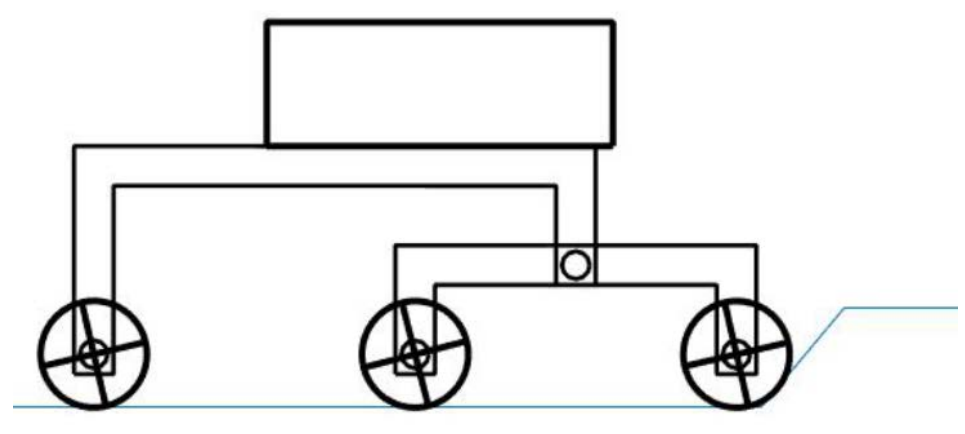

(a) Contact established

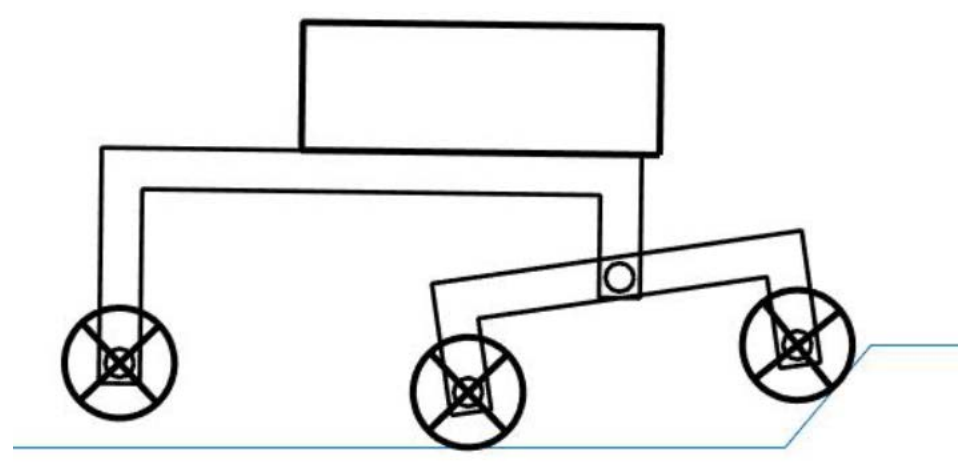

(b) Subsequent motion

Figure 5: Predicted motion of the robot prototype; $\theta=50^{\circ}$.

modes and the admissible velocity indicator. Another interesting application of these indicators is in the mechanical design of wheeled robots. Parameters such as the position of the centre of mass of the chassis, bogie length and bogie height can be varied and the indicators can be used to produce designs which have increased mobility and experience lower impact forces during obstacle negotiation.

\subsection{Experiments}

The predictions obtained with the indicators regarding the climbing ability of the robot were confirmed by experiments with the prototype shown in Fig. 2 . The validity of $T_{c}^{-}$was already shown in [5] and [9] with simulations and experiments. Here we present experimental results to validate the admissible velocity as indicator of the subsequent motion after contacting an 


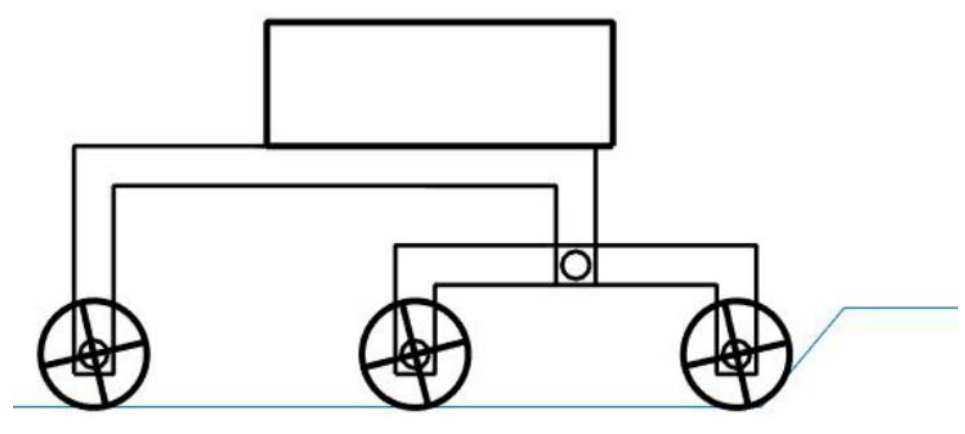

(a) Contact established

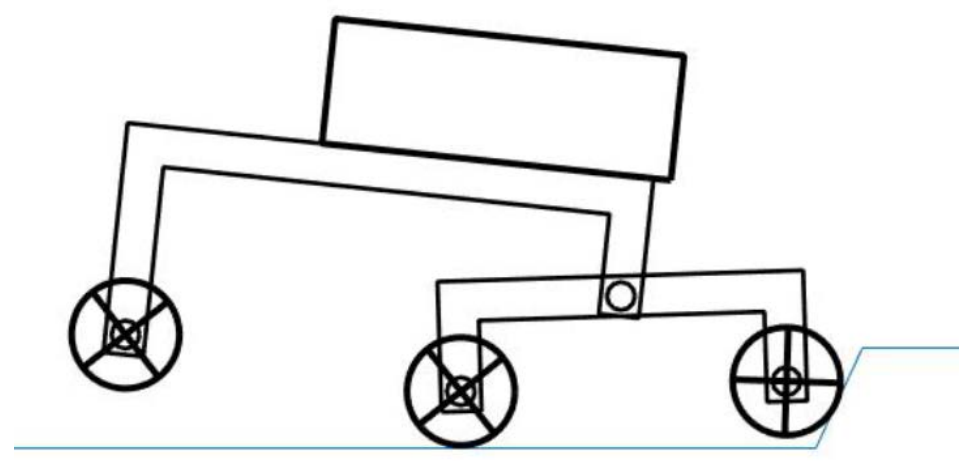

(b) Subsequent motion

Figure 6: Predicted motion of the robot prototype; $\theta=65^{\circ}$.

obstacle. In the experiment, the prototype was set to move towards a controllable-slope obstacle with a given initial velocity without wheel actuation. For the experiment shown in Figs. 7 and 8 the initial velocities were $1.2 \mathrm{~m} / \mathrm{s}$ and $1.0 \mathrm{~m} / \mathrm{s}$. The actual velocity is not that important because the aim was to qualitatively compare the modes of motion in the experiment with those indicated by the admissible velocity. No actuation was used to clearly show these modes of motion without additional effects. In Figs. 5 and 6 two modes of motion were predicted using the admissible velocity. At $\theta=50^{\circ}$ the robot started to climb the obstacle but at $\theta=65^{\circ}$ to robot climbed very little and tipped forward. The obstacle negotiation manoeuvre for $\theta=50^{\circ}$ is shown in Fig. 7 . The front wheels were able to overcome the obstacle, as predicted by the admissible velocity indicator. Fig. 8 shows the prototype behaviour for $\theta=65^{\circ}$; in this case, the front wheel came to a stop after contacting the obstacle. The modes of motion obtained in the experiment were qualitatively similar to those predicted by the admissible velocity indicator. 


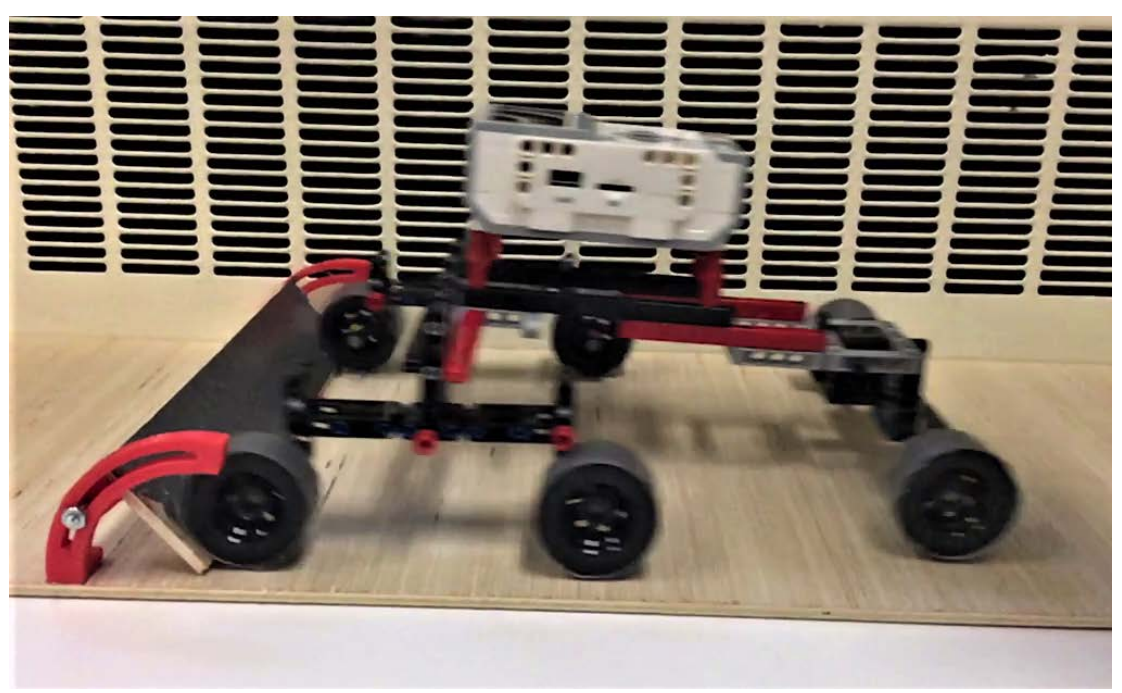

(a) Contact established

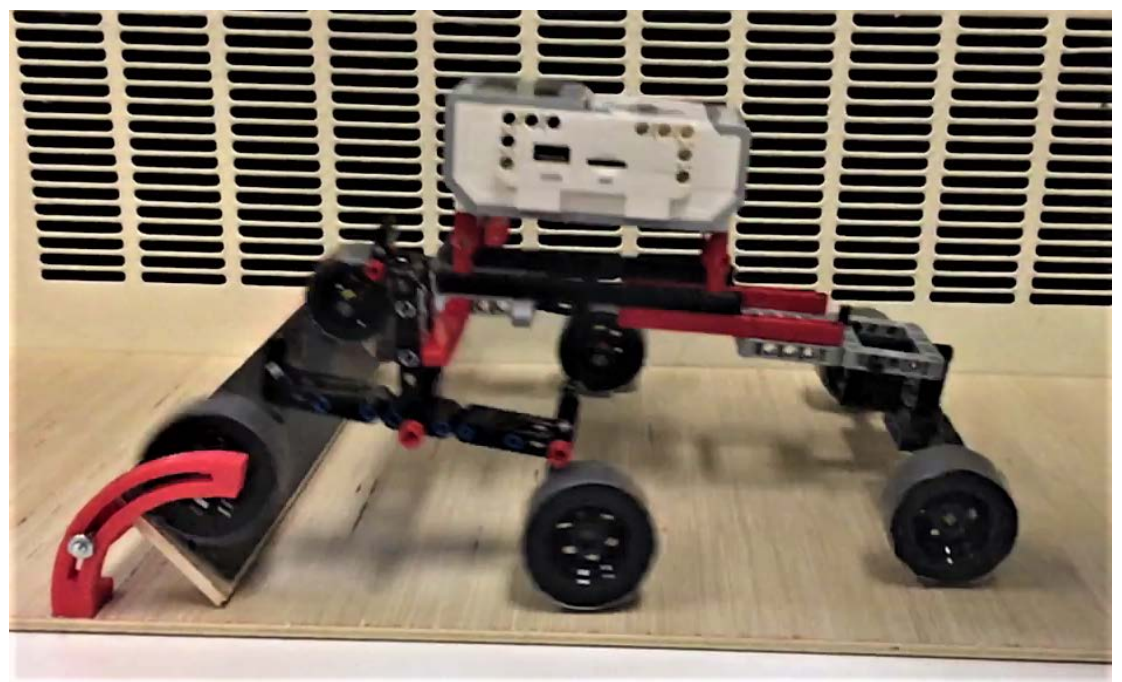

(b) Subsequent motion

Figure 7: Experimental motion of the robot prototype; $\theta=50^{\circ}$.

\subsection{Full-sized rover example}

The usefulness of the indicators was confirmed using as an example a full-sized lunar rover. The rover had a mass of $500 \mathrm{~kg}$ and featured a triple bogie suspension like the previous example. Its wheels had a radius of $0.24 \mathrm{~m}$. The distance between wheels was $1.0 \mathrm{~m}$. The centre of mass of its main body was $0.38 \mathrm{~m}$ above the centreline of its middle wheels. Finally, the front bogie pivot was $0.22 \mathrm{~m}$ above the centreline of its wheels.

The functional requirement was that this rover could traverse over the two obstacles shown in Fig. 9. In order to determine if the rover could meet this requirement, a forward-dynamics 


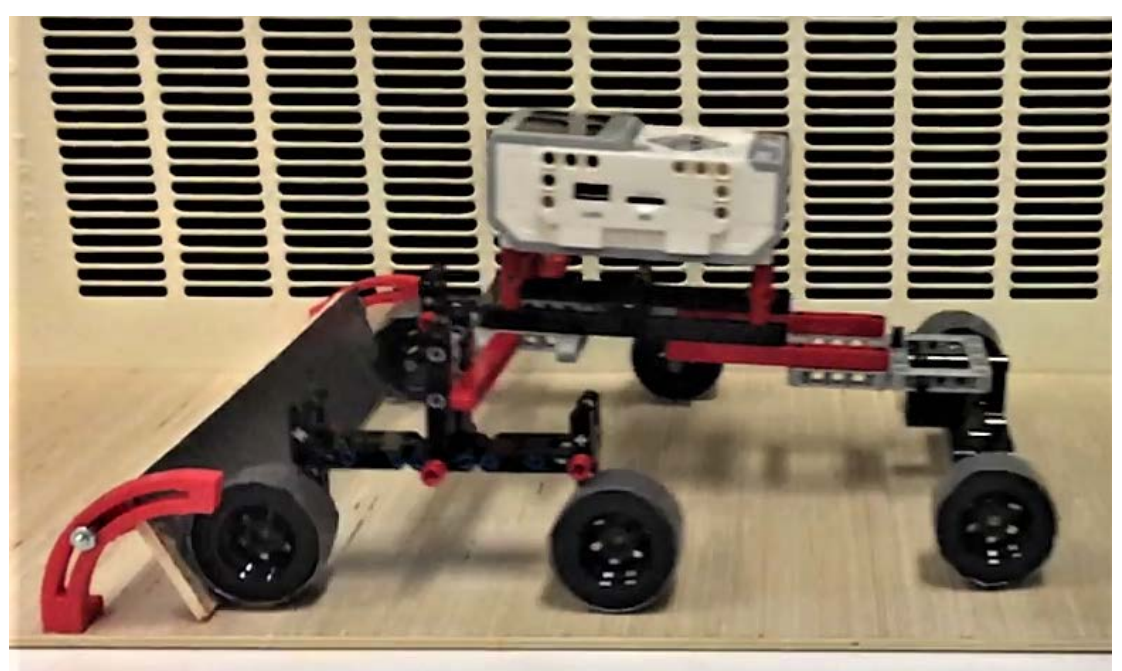

(a) Contact established

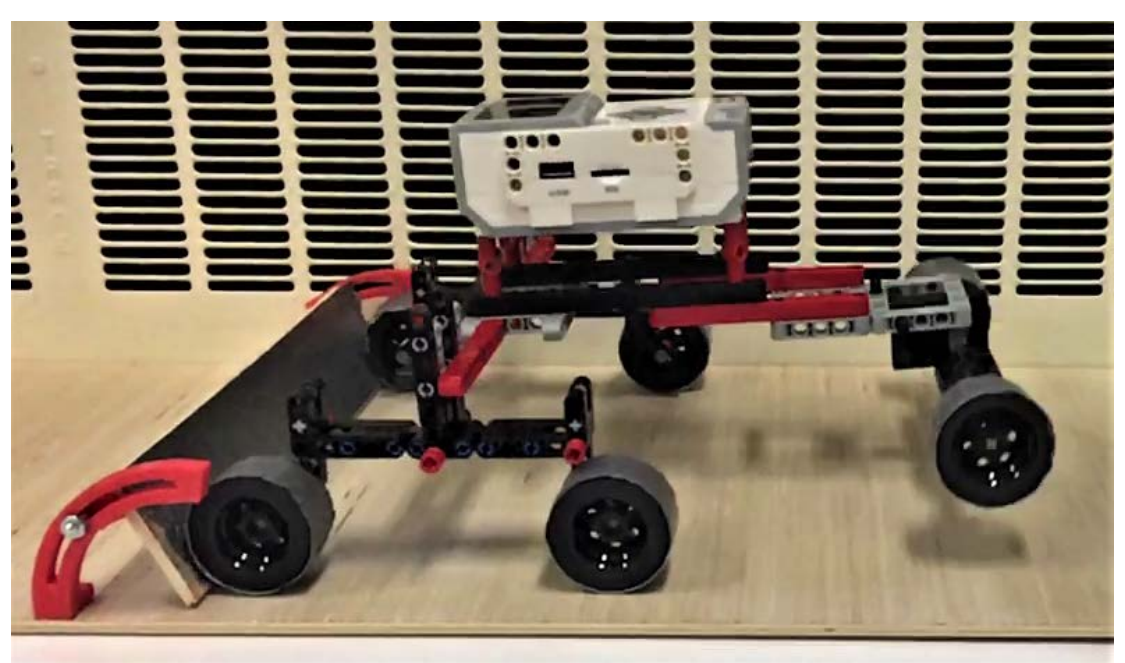

(b) Subsequent motion

Figure 8: Experimental motion of the robot prototype; $\theta=65^{\circ}$.

simulation model was developed in Matlab. The simulation was done by numerically integrating the equations of motion using the forward Euler method and $1 \mathrm{~ms}$ time steps. The equations of motion were expressed using a minimum set of two-dimensional coordinates. The normal contact forces between the wheels and ground or obstacle were computed using an explicit contact force model based on the penetration between the two bodies; a bristle friction model was used for the tangential contact forces [4].

In the first set of simulations, the rover was commanded to drive at a constant forward speed of $0.1 \mathrm{~m} / \mathrm{s}$ while traversing the obstacle. It was found that the friction coefficient was the most important parameter which determined whether or not the rover would succeed in the climbing manoeuvre. The traversal required a friction coefficient $\mu$ of at least 0.58 for obstacle 1 and 0.36 

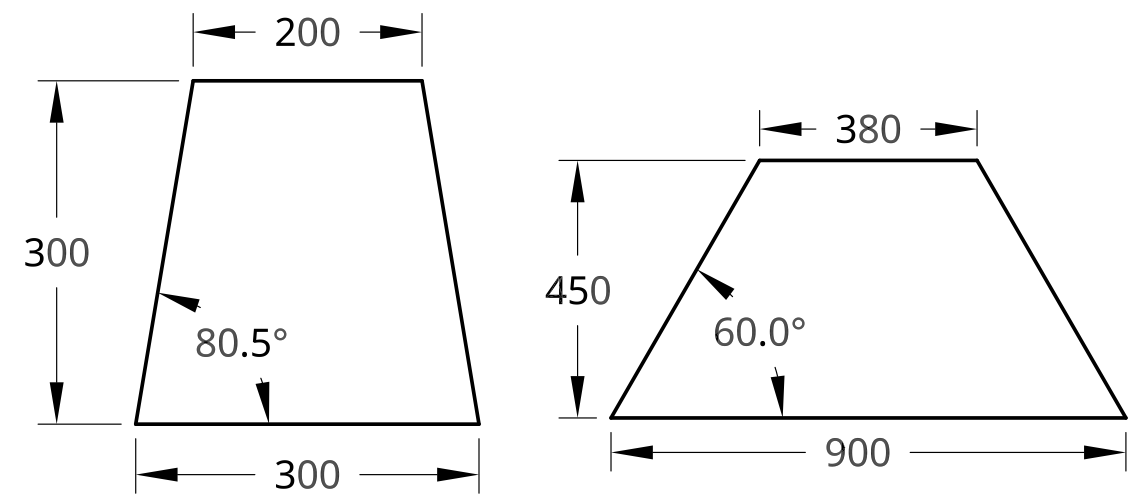

Figure 9: Obstacles 1 and 2. All dimensions are in $\mathrm{mm}$.

\begin{tabular}{|l|l|l|l|}
\hline & $T_{c}^{-}(\mathrm{J})$ & $T_{a}^{-}(\mathrm{J})$ & $v_{a}(\mathrm{~m} / \mathrm{s})$ \\
\hline front wheels & 2.24 & 0.49 & 0.00 \\
\hline middle wheels & 1.54 & 1.19 & 0.12 \\
\hline rear wheels & 1.51 & 1.22 & 0.10 \\
\hline
\end{tabular}

Table 1: Performance indicators for obstacle 1 assuming a forward speed of $0.1 \mathrm{~m} / \mathrm{s}$.

for obstacle 2 . The simulations of obstacle 1 showed that if the friction coefficient was lowered, e.g., to $\mu=0.5$, the front wheels would be able to cross the obstacle but not the middle wheels.

The performance indicators for the contact of each wheel with obstacle 1 can be found in Table 1. The admissible velocity for the front wheels is $0.0 \mathrm{~m} / \mathrm{s}$. This indicates that the front wheels tend to stop when they contact the obstacle. The mode of motion was found to be similar to the mode shown in Fig. 6 . Also note that most of the kinetic energy that the robot has immediately before contacting the obstacle will not contribute to overcoming it, but to increasing the impact force instead. Contrary to the front wheels, the admissible velocity for the middle and rear wheels is near the initial forward speed of $0.1 \mathrm{~m} / \mathrm{s}$. This means that after contacting the obstacle they will tend to go up along it. $T_{c}^{-}$is also lower for the middle and rear wheels indicating that the impact force would be lower when these wheels contact the obstacle compared to the front wheels. Using this information, we can hypothesize that increasing the speed of the rover before the middle and rear wheels contact the obstacle will increase its ability to overcome obstacle 1 . Additional simulation results showed that the rover could traverse obstacle 1 when the friction coefficient was set to $\mu=0.5$ if the vehicle speed was increased to $0.3 \mathrm{~m} / \mathrm{s}$ after the front wheels had overcome the obstacle. The increased speed helped the middle and rear wheels to bump up over the obstacle. The downside to increasing the vehicle speed is that the impact forces would be larger. In this case, $T_{c}^{-}$for the middle wheels would be $13.86 \mathrm{~J}$ which is nine times larger than 
the value in Table 1 since the rover is moving three times faster. A detailed structural analysis of the rover as well as information about the maximum shock that its instruments could endure would be required to determine if these impacts are acceptable.

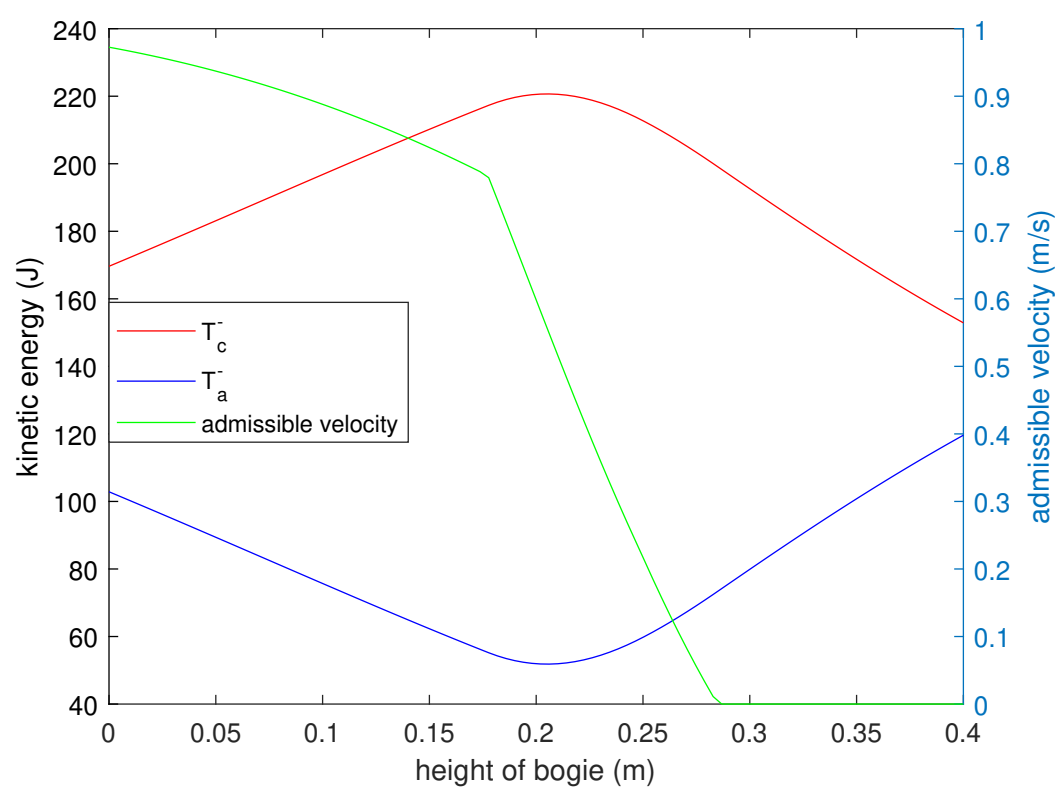

Figure 10: Variation of performance indicators with front bogie pivot height for the prototype lunar rover front wheels contacting an obstacle with a slope of 70 degrees at an initial speed of $1.0 \mathrm{~m} / \mathrm{s}$.

As this example showed, it is generally desirable that the admissible velocity indicator is as large as possible because it means that the momentum of the wheeled robot will aid it in overcoming an obstacle. Fig. 10 shows the variation of the performance indicators with the height of the front bogie pivot for the prototype lunar rover front wheels contacting an obstacle with a slope of $70^{\circ}$ at an initial speed of $1.0 \mathrm{~m} / \mathrm{s}$. This graph shows that the admissible velocity starts to rapidly decrease as bogie height increases beyond $0.18 \mathrm{~m}$ and is zero when the bogie height is above $0.29 \mathrm{~m} . T_{c}^{-}$also increases to a peak from $0 \mathrm{~m}$ to $0.21 \mathrm{~m}$ and then decreases because the rover starts to tip forward after that point. This shows that, for this particular problem, a low front bogie pivot height is desired to increase the tendency of the front wheels to climb an obstacle and to decrease impact forces.

To verify this, additional simulations of the rover rolling toward an obstacle with a 70-degree slope, at initial speeds of $0.5 \mathrm{~m} / \mathrm{s}$ and $1.0 \mathrm{~m} / \mathrm{s}$ were run to measure how high the front wheels would go up the obstacle for bogie joint heights ranging from $0.0 \mathrm{~m}$ to $0.4 \mathrm{~m}$. The results of these simulations are shown in Fig. 11. The graphs for both initial speeds have a similar shape with the difference being that at higher initial speed, the rover climbed higher up the obstacle. 


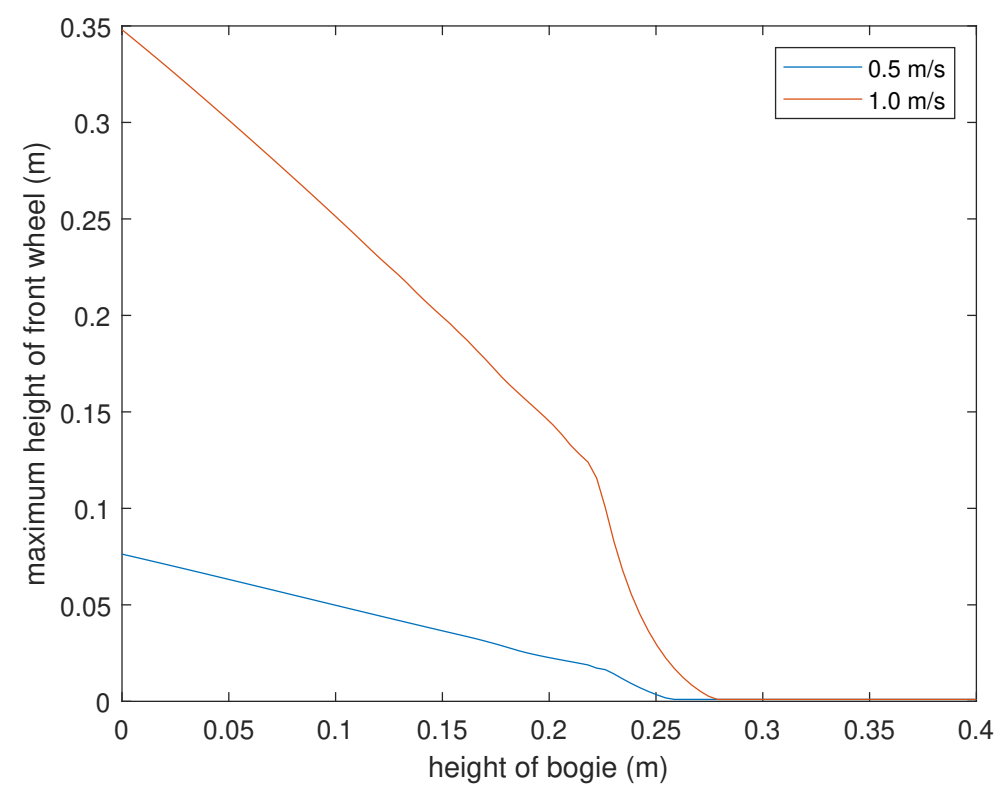

Figure 11: Maximum height reached by the front wheels of the rover up an obstacle with a $70^{\circ}$ slope vs. the height of the front bogie joint for initial velocities of 0.5 and $1.0 \mathrm{~m} / \mathrm{s}$.

These results show that the rover does not climb the obstacle when the bogie height exceeds $0.26 \mathrm{~m}$ at $0.5 \mathrm{~m} / \mathrm{s}$ and $0.28 \mathrm{~m}$ at $1.0 \mathrm{~m} / \mathrm{s}$. This was well predicted by the admissible velocities. The differences can be attributed to the fact that some assumptions are made about the contacts when computing $v_{a}$, as described in section 2 , whereas the simulations use an explicit contact model. The maximum height reached by the front wheels decreases near linearly with bogie pivot height. The admissible velocity gives an indication of the front wheel velocity immediately after the contact but it does not provide information about the subsequent time period when the rover is rolling up the obstacle. This is why the $v_{a}$ curve and the simulations results may have different shapes. $T_{a}^{-}$is an indicator of the portion of energy which remains unaffected by the impact but it does not indicate where that energy is going. In this case when the bogie pivot height is above $0.2 \mathrm{~m} T_{a}^{-}$increases but the front wheels do not climb up higher as a portion of the energy is spent in lifting the middle wheel.

\section{Conclusion}

This paper analyzed the mechanics of the first phase of the obstacle negotiation by a wheeled robot. A dynamics model and performance indicators were developed to characterize how the 
mechanical system of the robot can naturally influence its obstacle negotiation ability. We introduced the concepts of admissible mode of motion and admissible velocity, which describe how well the robot is expected to initiate the obstacle negotiation. The admissible mode is interpreted at the velocity level and reflects the effects of the mechanism design, mass and inertia distributions of the robot. The unilateral nature of the interaction among wheel, obstacle, and ground is also taken into account. The results of this work can be applied in the conceptual and detailed phases of the mechanical design of wheeled robots, the system level control design and path planning for terrain traversal, and in the operational analysis of robot missions.

\section{Acknowledgements}

This work was supported by the Natural Sciences and Engineering Research Council of Canada, and MacDonald, Dettwiler and Associates Space Missions. F. González was funded by the Ministry of Economy of Spain through the Ramón y Cajal programme, contract no. RYC-2016-20222.

\section{References}

[1] Apostolopoulos, D.: Analytical configuration of wheeled robotic locomotion. Ph.D. thesis, The Robotics Institute, Carnegie Mellon University (2001)

[2] Ghotbi, B., González, F., Kövecses, J., Angeles, J.: Mobility evaluation of wheeled robots on soft terrain: Effect of internal force distribution. Mechanism and Machine Theory 100, 259-282 (2016). DOI 10.1016/j.mechmachtheory.2016.02.005

[3] Gilardi, G., Sharf, I.: Literature survey of contact dynamics modelling. Mechanism and machine theory 37(10), 1213-1239 (2002)

[4] Gonthier, Y., McPhee, J., Lange, C., Piedboeuf, J.C.: A regularized contact model with asymmetric damping and dwell-time dependent friction. Multibody System Dynamics 11(3), 209-233 (2004) 
[5] González, F., Kövecses, J., Font-Llagunes, J.M.: Load assessment and analysis of impacts in multibody systems. Multibody System Dynamics 38(1), 1-19 (2016). DOI 10.1007/ s11044-015-9485-4

[6] Jarrault, C., Grand, B., Bidaud, C.: Large obstacle clearance using kinematic reconfigurability for a rover with active suspension. In: Proc. of the 13th Int. Conf. on Climbing and Walking Robots, pp. 114-121 (2010)

[7] Júdice, J.J.: Algorithms for continuous optimization, chap. Algorithms for linear complementarity problems, pp. 435-474. Springer (1994)

[8] Kövecses, J.: Dynamics of mechanical systems and the generalized free-body diagram part I: General formulation. Journal of Applied Mechanics 75(6, paper 061012), 1 - 12 (2008). DOI 10.1115/1.2965372

[9] MacMahon, S.: Modelling and contact analysis of planetary exploration rovers. Master's thesis, McGill University (2016)

[10] Papastavridis, J.G.: On the boltzmann-hamel equations of motion: A vectorial treatment. Journal of Applied Mechanics 61(2), 453 (1994). DOI 10.1115/1.2901466

[11] Patel, N., Slade, R., Clemmet, J.: The exomars rover locomotion subsystem. Journal of Terramechanics 47(4), 227-242 (2010)

[12] Skonieczny, K., D’Eleuterio, G.M.T.: Improving mobile robot step-climbing capabilities with center-of-gravity control. In: Proc. of the ASME 2010 International Design Engineering Technical Conferences, vol. 2, pp. 1531-1538 (2010). DOI 10.1115/DETC2010-28482

[13] Thueer, T., Siegwart, R.: Mobility evaluation of wheeled all-terrain robots. Robotics and Autonomous Systems 58(5), 508-519 (2010). DOI 10.1016/j.robot.2010.01.007 